\title{
Beyond the Star of Stars: an Introduction to Multi-Hop and Mesh for LoRa and LoRaWAN
}

\author{
Roger Pueyo Centelles, Felix Freitag, Roc Meseguer and Leandro Navarro
}

\begin{abstract}
LoRa has emerged in recent years as a wireless technology providing long-range communication for-low power Internet of Things (IoT) devices. The LoRaWAN architecture adds the Medium Access Control (MAC) and application layers and defines a star of stars topology suitable for many and diverse IoT applications. However, in a number of scenarios, a more flexible network topology than LoRaWAN's is needed. We review the state of the art of the design and implementation of multihop and mesh solutions for LoRa and LoRaWAN. We discuss the remaining challenges for multi-hop and mesh solutions to overcome in order to unlock the opportunities that decentralized, self-organizing and infrastructure-less LoRa networks can bring to IoT applications.
\end{abstract}

Index Terms-LoRa, LoRaWAN, mesh, multi-hop, IoT.

\section{INTRODUCTION}

A NUMBER of technologies providing wireless communications for IoT devices in a variety of scenarios have emerged during the last decade [1]. Among them, LoRa has proven successful in the Low Power Wide Area Network (LPWAN) domain for a vast number of deployments in diverse environments as a means to transmit small amounts of data over long distances. This radio technology is designed to operate in the sub-gigahertz range of the spectrum and employs Chirp Spread Spectrum (CSS) modulation, resistant to multi-path fading and suitable for noisy environments. It provides a throughput of up to tens of kbps, reaching beyond $10 \mathrm{~km}$ (outdoors, in rural areas), with low power consumption. Its characteristics have been thoroughly analyzed [2], [3], [4]. Manufacturers currently provide development boards with LoRa transceivers for prototyping and experimentation, as well as off-the-shelf encapsulated devices integrating sensors and radio, ready for deployment.

LoRa can be compared with the IEEE 802.15.4g standard for Wireless Smart Utility Networks. The former usually provides a longer transmission range, while the latter trades distance for higher throughput and is multi-hop capable. Communication ranges of $200 \mathrm{~m}$ (urban) and $800 \mathrm{~m}$ (open environment) are reported for 802.15.4g [5].

LoRaWAN is an open standard by the LoRa Alliance that defines the MAC and application layers and specifies an LPWAN protocol on top of LoRa. The architecture is designed to provide secure wireless bi-directional communication between end nodes and the application that processes the data.

This work was partially funded by the Spanish Government under contracts PID2019-106774RB-C21, PCI2019-111850-2 (DiPET CHIST-ERA), PCI2019-111851-2 (LeadingEdge CHIST-ERA), and the Generalitat de Catalunya as Consolidated Research Group 2017-SGR-990.

Manuscript received May 7, 2021.
Its star of stars topology, with the gateways at the center and the end nodes around them, has proven suitable for many and diverse IoT applications [6].

There are use cases, however, that would benefit from a more flexible topology than that of LoRaWAN to expand network coverage, overcoming the need for additional infrastructure, or to avoid sending data to the cloud in underserved locations. This paper elaborates on three representative scenarios that, either based on the LoRaWAN architecture or using plain LoRa, demand additional features like multihop, message broadcasting, device-to-device communication, routing or infrastructure-less operation. These features go beyond the scope of typical LoRaWAN and LoRa deployments, but researchers have already started to work on them. Their contributions range from simple unidirectional packet relaying designs to routing protocols that take advantage of LoRa's unique features, although they often only tackle particular problems. We match scenarios and proposals, analyzing which requirements have been addressed and what the remaining challenges are for multi-hop and mesh LoRa solutions to bring decentralized, self-organizing, and infrastructure-less networks to pervasive IoT systems.

\section{SCENARIOS BEYOND THE STAR OF STARS}

In LoRaWAN deployments, with a star of stars topology, gateways define the network's coverage area. This imposes an unbalanced uplink data path (nodes $\rightarrow$ gateway $\rightarrow$ cloud) that conditions the applications built upon. However, there are use cases that do not completely fit into this architecture and would benefit from more flexible network topologies. Some applications could be deployed with lower ownership costs by adopting network models that allow multi-hop packet transmission between nodes. This could be ultimately leveraged by infrastructure-less and decentralized IoT systems that need to distribute data among nodes scattered over large areas, connected by a low-power mesh network, to perform computations at the edge [7]. In the following, we analyze a number of these applications, including the state-of-the-art applications discussed below in Section III. We go on to classify them into three categories and illustrate them with representative use cases that demand multi-hop or mesh features.

\section{A. Extending coverage beyond the infrastructure reach}

While LoRa transmissions can cover long distances, it is difficult to provide LoRaWAN coverage for moving elements (e.g., vehicle fleets) circulating through vast areas (e.g., the open sea). There, deploying gateways can be economically 


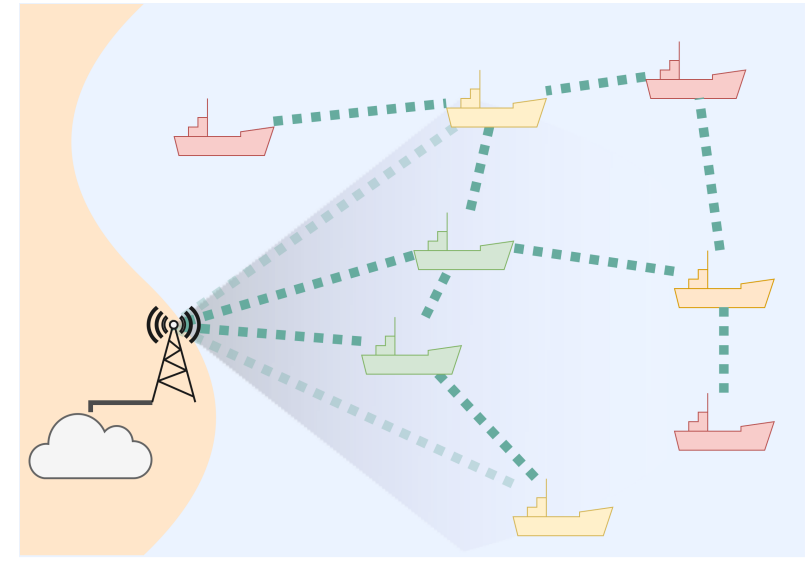

Fig. 1. A LoRaWAN-based use case where multi-hop could help extending the gateway coverage. The orange and red ships are out of the gateway's reach, but their messages could be relayed to the gateway by one of the green ships. Additionally, yellow ships could achieve better packet delivery ratios.

or technically challenging. In these environments, multi-hop packet transmission between end nodes could facilitate communication with gateways beyond their coverage.

Figure 1 depicts a real use case where a fleet of ships from a fishing guild needs to be tracked beyond the coverage of a mainland gateway. Although technically possible, deploying additional gateways is not a feasible option for the guild. However, the combination of multi-hop message transmission and Delay-Tolerant Network (DTN) can mitigate the effect of dark coverage areas and increase the network operation range. By leveraging the encounters of mobile elements and the new transmission paths they create, vessels closer to the shoreline can contribute to extending the gateway's coverage further offshore.

\section{B. Fault-tolerant systems}

In the aftermath of natural disasters, communication systems often experience downtime periods due to damage to the infrastructure (e.g., base stations) in the affected area. Under these conditions, the gateways in an IoT (specifically, LoRaWAN) network can become single points of failure and render a part of, or even the whole, network inactive if they cease to operate. To circumvent the failing infrastructure, direct communication between end nodes could be used.

Figure 2 shows the architecture of a communication system for the aftermath of an earthquake. In this envisioned scenario, people have small battery-powered devices equipped with a LoRa transceiver at their homes and workplaces. In case of emergency, they can use these devices to report their status to rescue teams or send short messages to their relatives. Gateways spread over the city receive the messages and forward them to the emergency coordination workforce. If the LoRaWAN gateways are rendered isolated or inoperative by adverse conditions, user nodes could take a proactive role in the system and help to route packets from and to those nodes affected by infrastructure failures.

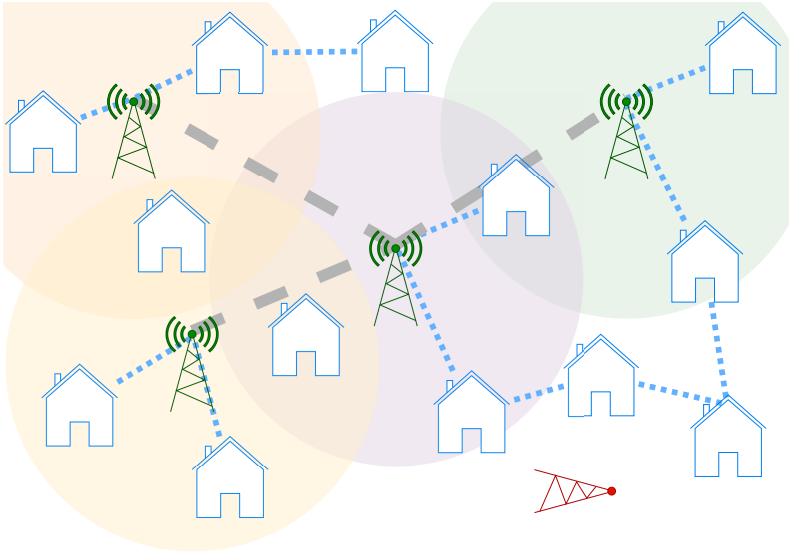

Fig. 2. After a natural disaster, like an earthquake, some infrastructures may become unavailable. End nodes with the ability to communicate between themselves can forward messages to the remaining data sinks (and back).

This scenario has fundamental differences with the previous one (described in Section II-A): it has a much higher number of nodes, which requires an efficient and coordinated use of the spectrum, and an infrastructure that can unpredictably fail when it is most needed. On the other hand, no mobility is expected.

\section{Infrastructure-less systems}

Obtaining metering data in the field is a slow, laborintensive, and expensive task. Many cities worldwide, and even entire countries, have digitized readings from public utilities, etc., using wireless or Power Line Communications (PLC). LoRa can be used to facilitate this process in underserved areas if transceivers are embedded into metering devices. Applications in this domain could also adopt mesh topologies instead of the gateway-centric model. Nodes could relay messages from one metering device to another until they reach a data sink and also in the opposite direction. This can be of particular interest in deployments with low density of nodes, where a low nodes/gateway ratio may be economically impractical.

Figure 3 shows a real use case: a drinking water utility that spreads over a vast remote mountainous region. Currently, manual intervention is required to register water quality at different settlements. Due to insufficient telecommunication infrastructure, obtaining and reporting the data is timeconsuming and expensive. The measurements are being automated to improve management efficiency, but the measurement apparatus must be linked to a communication system that can report data to distant facilities. The LoRaWAN architecture does not fit this scenario, as the size of the deployment and the geography of the terrain would require a high number of gateways. As most of the nodes are in the line of sight of other nodes, a multi-hop solution could instead be employed, forwarding packets between the different settlements until they reach the management facilities. 


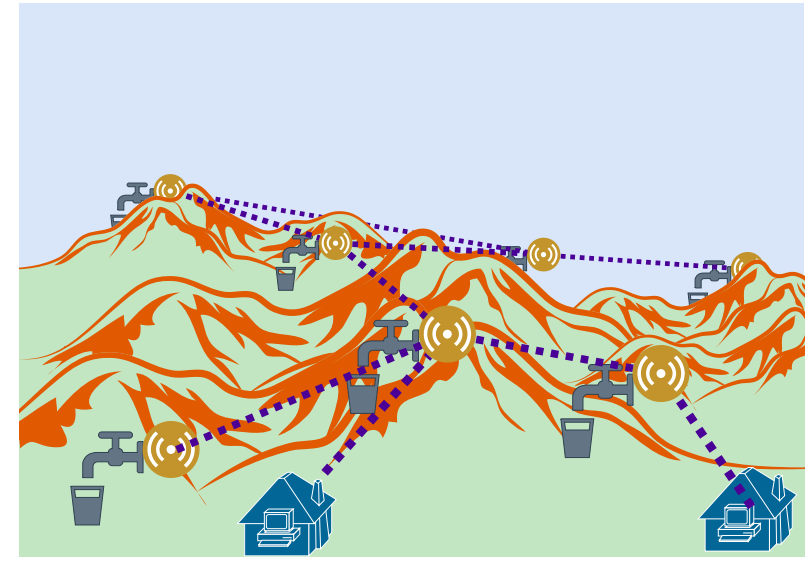

Fig. 3. Sparsely-distributed sensors cannot be easily covered by LoRaWAN gateways. However, by leveraging multi-hop communications, data can be relayed node to node until reported to the management facilities.

\section{STATE OF THE ART}

Researchers have already started to design and implement multi-hop and mesh solutions with LoRa and LoRaWAN for diverse purposes, with different degrees of complexity and completeness. We surveyed them, picked the most relevant ones (namely, those with some kind of experimental evaluation), and classified them into five categories, based on their aims. Here we discuss their objectives and features, showing how they can help in the development of more flexible network topologies. Table I summarizes these contributions.

\section{A. Extending LoRaWAN gateways coverage}

The first objective pursued is extending the coverage of LoRaWAN deployments beyond the gateways' reach, matching the scenario from Section II-A. The motivation for increasing the coverage usually comes as a consequence of technical or economic barriers to the installation of additional gateways, such as a lack of suitable locations, inadequate electricity or Internet connectivity, and associated costs. Infrastructure ownership can also play an important role: end users and infrastructure providers might be different entities with different interests.

Dias and Grilo designed and implemented an uplink multihop solution to extend the coverage of LoRaWAN gateways [8]. Arguing that deploying additional gateways is not always an option (for instance, when users are not infrastructure owners), they suggest deploying intermediate nodes that relay data packets from end nodes to gateways. Their proposal contains a simplified version of the DestinationSequenced Distance Vector (DSDV) Routing Protocol (RP), running on intermediate Routing Nodes, coordinated to relay uplink packets to the best available gateway. This approach could additionally mitigate infrastructure failures since relay nodes would be able to forward messages to the closest available gateway. The solution is compatible with existing LoRaWAN deployments but only covers uplink transmissions from end nodes operating in Activation by Personalization (ABP) mode.
Among other challenges, Sartori et al. addressed the gateways' coverage issue and designed RLMAC, a MAC layer protocol that enables Routing over Low Power and Lossy Networks (RPL) multi-hop communications based on LoRa [9]. They argue that the star topology is convenient for ease of deployment and from a business perspective, though multi-hop could mitigate congestion issues. Moreover, multi-hop could be the only option for covering very large areas with few base stations and could increase throughput or reduce timeon-air by using faster Spreading Factors (SFs). The authors designed a multi-hop solution for single-channel LoRa nodes. They implemented the algorithms to bootstrap and operate a network using RPL by combining a slow reception loop with fast transmission loops, ensuring that nodes can receive messages using any SFs.

Lundell et al. designed a routing protocol to provide mesh networking between gateways to extend coverage [10]. The authors claim that, in both urban and rural scenarios, gateways without Internet access could forward packets towards those with a backhaul connection. They took Hybrid Wireless Mesh Protocol (HWMP) and Ad-Hoc On-Demand Distance Vector (AODV) as their starting point, adapting them to the specifics of LoRa, and built a packet tunneling mechanism. Their protocol is transparent to both ends (nodes and LoRaWAN server) and was validated in laboratory experiments and field tests, with uplink messages traversing a 4-hops network. Downlink transmission was considered, but not tested.

\section{B. Multi-hop linear networks}

Monitoring systems for linear utilities that connect distant points (power lines, waterways, piping systems, etc.) are common among IoT deployments. While LoRa provides long transmission distances, it may not suffice for systems spanning over hundreds of kilometers where gateways need to be deployed at intervals along the utility. Similarly, underground deployments in sewage systems, mines, etc., experience equivalent coverage issues. While this category could be viewed as only a particular case of the previous one in Section III-A, authors have provided solutions to address the problems in these specific conditions.

Duong and Kim designed and implemented a protocol with multi-hop communication for LoRa networks covering large distances [11]. Their solution was intended for deployments where every monitoring node is placed along a line, such as a gas pipe or a high voltage line. The nodes forward data in the leaf $\rightarrow$ sink direction. Devices are synchronized and wake up at specific moments in time to receive data packets from their neighbors, which they can combine with their own data packets and send further along the line. Downlink data transmission is not covered.

Similarly, Abrardo and Pozzebon designed a multi-hop LoRa linear network for underground environments, optimizing the nodes' sleep/wake cycles to reduce battery consumption [12]. They found that LoRa transmission was limited to around $200 \mathrm{~m}$, making the star topology unsuitable for the pervasive monitoring of very long aqueducts with curves that obstruct line of sight. To overcome this limitation, they opted 
for a data propagation model with sensor nodes forming a transmission chain towards the gateway, including a synchronization mechanism when propagating data between pairs of nodes to maximize the sleep cycles' duration.

\section{Gateway-less, cloud-less deployments}

Another common objective we identified is to replace the centralized gateways and cloud server infrastructure with inthe-premises, self-managed systems that harness computation at the edge, enabling distributed, fully decentralized applications. Motivations for this are diverse, ranging from technical requirements (e.g., distributed applications where the centralized data sink design does not apply because end-node devices better integrate the control loop of certain IoT requirements using a gateway-less mesh network) to data privacy, security, and sovereignty (i.e., independence of the network deployment from external providers).

Lee and $\mathrm{Ke}$ designed and implemented a LoRa mesh networking system to ensure that indoor nodes can communicate with network servers without deploying more gateways [13]. Their design consists of a data sink (rather misleadingly, the authors call it a gateway) broadcasting beacons to invite nodes to join the network. Those, in turn, set the gateway (i.e., the data sink) as their parent. New nodes that hear packets from the gateway, or other nodes, can also join the network, choosing a suitable parent based on multiple factors (namely, Received Signal Strength Indicator (RSSI), hop count). The gateway polls children nodes to request their data and holds a complete view of the network topology, which it can modify based on its comprehensive information. The authors state that, while their solution extends the coverage of a network without installing more gateways, the number of serviced nodes would be smaller than with a conventional star topology because of the latency introduced by successive packet forwarding.

Kim et al. proposed an Adaptive Spreading Factor Selection (ASFS) scheme to build LoRa mesh networks using singlechannel transceivers, increasing throughput and reducing costs [14]. Their proposal uses the modems' Channel Activity Detection (CAD) capability with an iterative SF inspection and selection algorithm that allows links to operate independently at different data rates, achieving almost $100 \%$ correct detection. This idea had been implemented already on single-channel gateways but had not previously been adapted for multi-hop usage. The authors experimentally evaluate the proposal with up to 10 nodes and compare three topologies (star, tree, and mesh) using Semtech's single-channel SX1272 and multi-channel SX1301 transceivers. Using ASFS allows nodes to choose different and faster SFs, achieving data rates four to six times faster than without it (when all the nodes stick to a common, network-wide slower SF).

\section{Energy efficiency-aware network}

The SF is a key parameter of the CSS LoRa modulation, since it determines a trade-off between transmission distance and bandwidth. Higher SF values and, hence, longer distances come at the expense of longer time-on-air: each step up in the SF doubles the time required to transmit the same data, also doubling energy consumption. For this reason, certain use cases can benefit from shorter multi-hop transmissions using lower SFs that distribute energy consumption more evenly among nodes.

Nunez Ochoa et al. analyzed different LoRa radio parameters (SF, bandwidth, transmission power) and computed the energy consumption of the transceivers for both star and mesh topologies [15]. They analyzed the flexibility LoRa offers to configure its radio parameters for different deployment scenarios, considering its impact on the energy consumption of radio transmission and reception. They proposed various strategies to reduce this energy consumption. For a mesh topology, energy consumption was optimized by applying different radio configurations for different network layouts, where the density of nodes played a determinant role in coverage and number of hops.

\section{E. Decentralized, flat mesh deployments}

The latest step in the pursuit of more flexible network topologies than the star of stars is the development of multihop protocols to build mesh networks, in which nodes coordinate in a decentralized manner. This way, the gateway-end nodes hierarchy is flattened, resulting in horizontal network deployments. There, all the nodes may have equal duties regarding network operation, network organization, and traffic forwarding.

McCauley developed RadioHead, a packet radio software for embedded processors [16]. It provides an object-oriented library for sending and receiving messages via a variety of data radio technologies, including LoRa. In particular, it contains the RHMesh subclass for sending addressed, optionally acknowledged, datagrams across a network using multi-hop. The class adds automatic route discovery and route signaling within a mesh of adjacent nodes. It can be used in networks with dynamic topologies, where nodes move around or become unavailable. RHMesh uses reliable hop-to-hop message delivery, but not end-to-end acknowledgments. The class does not have message queuing, meaning that it can only handle one message at a time.

Hester and several other contributors are working on Meshtastic [17], a project for using inexpensive development boards with GPS, battery and a LoRa chip as secure mesh communicators. Meshtastic is intended for outdoor sport activities or any other situation with no Internet access. Users create a private mesh to exchange their location and send text messages to a group chat. Devices forward packets using a flooding algorithm to reach the furthest member. The chosen hardware is based on a WiFi/BLE-capable ESP32 System on a Chip (SoC) bundled with a LoRa transceiver and, optionally, a GPS receiver.

Pycom provides commercial development boards and OEM products for IoT projects in the Python language. These devices can run Pymesh, a firmware for flexible LoRa mesh networking [18]. It provides encrypted ad-hoc communication over raw LoRa, implements Listen-before-talk (LBT) MAC, and supports multiple node roles (leader, router, child, and border router). The firmware also has some routing capabilities, as it claims to forward packets via the best link available. 
TABLE I

SUMMARY OF THE SURVEYED MULTIHOP AND MESH SOLUTIONS FOR LORA AND LORAWAN

\begin{tabular}{|c|c|c|c|c|c|c|}
\hline Authors & $\begin{array}{l}\text { Challenges } \\
\text { addressed }\end{array}$ & $\begin{array}{l}\text { Application } \\
\text { scenario }\end{array}$ & Approach & Validation & Potential & Limitations \\
\hline $\begin{array}{lr}{[8]} & \text { Dias } \\
\& & \text { Grilo } \\
(2018) & \end{array}$ & $\begin{array}{l}\text { Coverage extension } \\
\text { without adding more } \\
\text { gateways }\end{array}$ & $\begin{array}{l}\text { Dark spots in urban } \\
\text { deployments }\end{array}$ & $\begin{array}{lr}\text { Intermediate } & \text { relay } \\
\text { nodes implementing } \\
\text { simple r DSDV } \\
\text { routing }\end{array}$ & $\begin{array}{l}\text { Prototype } \\
\text { assessment with } \\
4 \text { routing nodes }\end{array}$ & $\begin{array}{l}\text { Up-link extension } \\
\text { of multi-stakeholder } \\
\text { LoRaWAN network }\end{array}$ & $\begin{array}{l}\text { No down-link trans- } \\
\text { missions }\end{array}$ \\
\hline $\begin{array}{lr}\text { [9] } & \text { Sartori } \\
\text { et } & \text { al. } \\
(2017) & \\
\end{array}$ & $\begin{array}{l}\text { Large areas coverage } \\
\text { with few base sta- } \\
\text { tions }\end{array}$ & $\begin{array}{lr}\begin{array}{lr}\text { Distributed } \\
\text { isolated } \\
\text { deployments }\end{array} & \text { or } \\
\end{array}$ & $\begin{array}{l}\text { Extension of RPL } \\
\text { with new OF and } \\
\text { metrics }\end{array}$ & $\begin{array}{l}\text { Building-sized } \\
\text { experimental setup } \\
\text { with } 5 \text { nodes }\end{array}$ & $\begin{array}{l}\text { Infrastructure-less } \\
\text { IoT, in-the-premises } \\
\text { computing }\end{array}$ & $\begin{array}{l}\text { Unbalanced network, } \\
\text { bottleneck in RPL } \\
\text { root nodes }\end{array}$ \\
\hline $\begin{array}{l}{[10] \text { Lun- }} \\
\text { dell et al. } \\
(2018)\end{array}$ & $\begin{array}{lr}\text { Coverage } & \text { extension } \\
\text { without } & \text { Internet } \\
\text { backhaul } & \end{array}$ & $\begin{array}{l}\text { Large rural sensors } \\
\text { networks, urban IoT } \\
\text { deployments }\end{array}$ & $\begin{array}{l}\text { Gateways mesh for } \\
\text { transparent node to } \\
\text { server tunneling }\end{array}$ & $\begin{array}{l}\text { Proof-of-concept im- } \\
\text { plementation with } 4 \\
\text { gateways }\end{array}$ & $\begin{array}{l}\text { Monitoring of very } \\
\text { large areas, gateway- } \\
\text { based backbone } \\
\text { mesh network }\end{array}$ & $\begin{array}{l}\text { No down-link trans- } \\
\text { missions }\end{array}$ \\
\hline $\begin{array}{l}\text { [11] Duong } \\
\& \text { Kim } \\
(2017)\end{array}$ & $\begin{array}{l}\text { Networks covering } \\
\text { long distances in a } \\
\text { linear topology }\end{array}$ & $\begin{array}{l}\text { Monitoring of linear } \\
\text { utility deployments }\end{array}$ & $\begin{array}{l}\text { Linear leaf to sink } \\
\text { multihop packet for- } \\
\text { warding }\end{array}$ & $\begin{array}{l}\text { Campus-sized exper- } \\
\text { imental setup with } 5 \\
\text { nodes }\end{array}$ & $\begin{array}{l}\text { Management of } \\
\text { long-distance, } \\
\text { linear topology } \\
\text { infrastructure }\end{array}$ & $\begin{array}{l}\text { Unidirectional com- } \\
\text { munication, reduced } \\
\text { PDR and throughput }\end{array}$ \\
\hline $\begin{array}{l}\text { [12] Abrardo } \\
\& \\
\text { Pozzebon } \\
(2019)\end{array}$ & $\begin{array}{l}\text { Underground } \\
\text { networks } \\
\text { limited range }\end{array}$ & $\begin{array}{l}\text { Monitoring of } \\
\text { tunnels and } \\
\text { subterranean utilities }\end{array}$ & $\begin{array}{l}\text { Linear origin to sink } \\
\text { multihop packet for- } \\
\text { warding }\end{array}$ & $\begin{array}{l}\text { In-place } \\
\text { measurements, } \\
\text { laboratory tests, } \\
\text { analytical analysis }\end{array}$ & $\begin{array}{l}\text { Management of } \\
\text { underground, } \\
\text { linear topology } \\
\text { infrastructure }\end{array}$ & $\begin{array}{l}\text { Unidirectional com- } \\
\text { munication, nodes } \\
\text { synchronization }\end{array}$ \\
\hline $\begin{array}{l}\text { [13] Lee et } \\
\text { al. (2018) }\end{array}$ & $\begin{array}{l}\text { Indoor nodes' com- } \\
\text { munication in dense } \\
\text { urban areas }\end{array}$ & $\begin{array}{l}\text { Campus-sized moni- } \\
\text { toring environment }\end{array}$ & $\begin{array}{l}\text { Mesh system, RSSI } \\
\text { and hop-count-based } \\
\text { next-hop selection }\end{array}$ & $\begin{array}{l}\text { is-sized exper- } \\
1 \text { testbed with } \\
\text { les }\end{array}$ & $\begin{array}{l}\text { Collaborative LoRa } \\
\text { mesh networks for } \\
\text { very large areas }\end{array}$ & $\begin{array}{l}\text { Smaller density than } \\
\text { with a star topology }\end{array}$ \\
\hline $\begin{array}{lr}{[14]} & \text { Kim } \\
\text { et } & \text { al. } \\
(2020) & \\
\end{array}$ & $\begin{array}{l}\text { Throughput } \\
\text { enhancement for } \\
\text { single-channel nodes }\end{array}$ & High traffic networks & $\begin{array}{lr}\begin{array}{l}\text { Adaptive } \\
\text { Factor }\end{array} & \text { Spreading } \\
\text { (ASFS) } & \\
\end{array}$ & $\begin{array}{l}\text { Campus-sized exper- } \\
\text { imental testbed with } \\
10 \text { nodes }\end{array}$ & $\begin{array}{l}\text { Overlaid, simultane- } \\
\text { ous multi-SF opera- } \\
\text { tion }\end{array}$ & $\begin{array}{l}\text { Complex routing al- } \\
\text { gorithm required for } \\
\text { best results }\end{array}$ \\
\hline $\begin{array}{l}{[15] \text { Nunez }} \\
\text { Ochoa } \\
\text { (2017) }\end{array}$ & $\begin{array}{l}\text { Energy consumption } \\
\text { optimization }\end{array}$ & $\begin{array}{lr}\text { Very } & \text { energy- } \\
\text { constrained } & \text { end } \\
\text { nodes } & \end{array}$ & $\begin{array}{l}\text { Combination of star } \\
\text { and mesh topologies }\end{array}$ & Analytic calculations & $\begin{array}{l}\text { Lifetime extension } \\
\text { of battery-operated } \\
\text { nodes }\end{array}$ & $\begin{array}{lr}\text { Real-life retworks' } \\
\text { dynamics, rack } \\
\text { of experimental } \\
\text { validation } \\
\end{array}$ \\
\hline $\begin{array}{l}{[16] \text { Mc- }} \\
\text { Cauley } \\
(2014)\end{array}$ & $\begin{array}{l}\text { Datagrams transmis- } \\
\text { sion over a multihop } \\
\text { network }\end{array}$ & $\begin{array}{lr}\text { Dynamic } & \text { networks } \\
\text { with } & \text { varying } \\
\text { topology } & \end{array}$ & $\begin{array}{l}\text { Automatic route dis- } \\
\text { covery and signaling }\end{array}$ & Software library & $\begin{array}{ll}\text { Gateway- } & \\
\text { less, } & \text { context- } \\
\text { aware } & \text { network } \\
\text { deployments } & \\
\end{array}$ & $\begin{array}{l}\text { Missing end-to-end } \\
\text { ACK and message } \\
\text { queuing }\end{array}$ \\
\hline $\begin{array}{l}\text { [17] Hester } \\
\text { et } \quad \text { al. } \\
(2020)\end{array}$ & $\begin{array}{l}\text { Very long range data } \\
\text { broadcast }\end{array}$ & $\begin{array}{l}\text { Off-grid, outdoors, } \\
\text { emergency } \\
\text { communication } \\
\text { and location }\end{array}$ & $\begin{array}{l}\text { Broadcast smart data } \\
\text { flooding }\end{array}$ & $\begin{array}{l}\text { Available, off-the- } \\
\text { shelf devices }\end{array}$ & $\begin{array}{l}\text { Autonomous, off- } \\
\text { grid communication } \\
\text { for communities }\end{array}$ & $\begin{array}{l}\text { Scalability, no rout- } \\
\text { ing }\end{array}$ \\
\hline $\begin{array}{l}{[18] \quad \text { Py- }} \\
\text { com } \\
(2020)\end{array}$ & $\begin{array}{l}\text { Network flexibility, } \\
\text { decentralization }\end{array}$ & $\begin{array}{l}\text { Self-organizing, } \\
\text { multi-gateway mesh } \\
\text { networks }\end{array}$ & $\begin{array}{lr}\text { Routing } & \text { protocol, } \\
\text { multiple } & \text { network } \\
\text { roles } & \end{array}$ & $\begin{array}{l}\text { Development } \\
\text { devices } \\
\text { commercially } \\
\text { available }\end{array}$ & $\begin{array}{lr}\text { OEM } & \text { integration } \\
\text { in } & \text { off-the-shelf } \\
\text { products } & \text { and } \\
\text { applications } & \\
\end{array}$ & $\begin{array}{l}\text { Closed source, } \\
\text { proprietary solution, } \\
\text { incompatibility with } \\
\text { other vendors }\end{array}$ \\
\hline $\begin{array}{l}19] \text { NiceRF } \\
(2020)\end{array}$ & $\begin{array}{l}\text { Serial data } \\
\text { communication } \\
\text { over encrypted mesh }\end{array}$ & $\begin{array}{l}\text { Remote control, } \\
\text { telemetry, } \\
\text { automation }\end{array}$ & $\begin{array}{l}\text { Multiple } \\
\text { roles, } \\
\text { protocol }\end{array}$ & $\begin{array}{l}\text { Readily available } \\
\text { commercial product }\end{array}$ & $\begin{array}{l}\text { Machine to machine } \\
\text { (M2M) communica- } \\
\text { tions }\end{array}$ & $\begin{array}{l}\text { Closed source, } \\
\text { proprietary solution, } \\
\text { incompatibility with } \\
\text { other vendors }\end{array}$ \\
\hline
\end{tabular}

Unfortunately, Pymesh can only run on Pycom's products, making it incompatible with other vendors.

NiceRF commercially offers the SV-Mesh and LoRaStar range of LoRa transceivers. These products, available as embedded boards or packaged devices, provide serial TTL, RS232, or RS482 communication over LoRa links. They consist of a low power microcontroller and a regular LoRa transceiver. The manufacturer developed the proprietary LoRaPro mesh networking protocol, which defines a 2 byte addressing scheme, three network roles (node, router, node plus router), and a virtually unlimited number of routes.

\section{Discussion}

The review above reveals a variety of solutions achieving multi-hop communication for LoRa and LoRaWAN, ranging from simple packet relaying mechanisms to very complete proposals that include routing algorithms for manifold topologies, energy-efficient sleep/wake cycle management, etc. We introduce four performance indicators to evaluate the capabilities of the multi-hop and mesh proposals as compared to the LoRaWAN architecture. The diverse requirements of the scenarios presented in Section II are covered by the proposed solutions to different extents. We analyze each case to determine which challenges have been addressed.

\section{A. Performance indicators}

1) Throughput: The star of stars topology used in the LoRaWAN architecture imposes an unbalanced communication model that benefits uplink transmissions. Gateways receive packets from diverse nodes on different channels and SFs simultaneously, while nodes can only receive in one channel with one SF. Throughput of LoRaWAN networks has been thoroughly analyzed by different authors and depends on several factors (number of nodes and density, payload size, medium access method, etc.) [2], [3]. As a rule of thumb, each end node can attain an uplink throughput of $1.8 \mathrm{kbps}$, while each gateway can receive up to $27 \mathrm{kbps}$ per channel (both on the application data layer) [20].

Multi-hop and mesh solutions provide communication between all nodes and tend to balance traffic asymmetry. By 
distributing traffic more evenly, these solutions reduce the concentration effect of gateways. For example, the multi-hop network of Kim et al. covered an area of roughly $500 x 250 \mathrm{~m}^{2}$, comparable to a small LoRaWAN cell. Their experiments with 10 nodes achieved an aggregated throughput of $6.921 \mathrm{~kb} / \mathrm{s}$, equivalent to $25 \%$ of the expected throughput a gateway could provide. While performance depends on particular aspects of the deployment (topology, transmission scheduling, etc.), multi-hop and mesh solutions will generally trade-off throughput for flexibility.

2) Latency: LoRaWAN defines that nodes and gateways communicate directly using one hop transmissions. Assuming a packet transmission is correctly received by the gateway, its latency will be mainly the sum of the LoRa time-on-air, the reception and forwarding at the gateway, the trip to the network server (usually in the cloud) and the data processing by the application. In LoRaWAN multi-hop environments, extending the network coverage will come at the price of increased latency, due to the required time-on-air and any processing delays. For instance, Lundell et al.'s gateways mesh network adds artificial delays to avoid collisions, but also requires up to a few seconds for routes to be initially discovered [10].

In cloud-less mesh deployments, direct node-to-node communication avoids sending data through a gateway, to the Internet, and back. In some cases, this can save time for real-time applications [17], [14] but, in general, multi-hop will add flexibility in exchange for longer end-to-end delays. However, saturated links may become bottlenecks, as duty cycle restrictions could provoke significant delays in paths with several hops. Therefore, unless traffic is very scarce, careful route selection, synchronization, packet prioritization, or MAC mechanisms should be put into place.

3) Scalability: Researchers have extensively analyzed the scalability of LoRaWAN networks, finding that a single gateway can cover thousands of end nodes spread over some $\mathrm{km}^{2}$ if they transmit only few times per day [3], [20], [4]. This is mostly due to the superior reception capacity of the gateways' hardware. However, the surveyed multi-hop and mesh proposals have been evaluated with only a few tens of nodes [12], [13]. There, efficient network algorithms will be required to minimize the overhead (topology discovery, clustering, routing, etc.), as the growth in the number of nodes will be restrained by LoRa's limited bandwidth budget. These include channel hopping, multi-SF allocation, and MAC mechanisms, which some of the reviewed proposals only implement separately. Regarding the coverage area, however, the surveyed proposals indicate that multi-hop LoRa is a convenient strategy to scale up LPWANs [8], [10], [17].

4) Energy awareness: The LoRaWAN architecture defines three operation classes for end node devices: A, B and C. They all support bi-directional communication, but handle downlink traffic differently, allowing devices to save energy. End nodes typically belong to class A: they spend most of the time sleeping, wake up only to perform a measurement, transmit the datum uplink, and eventually receive a downlink packet. These devices can operate for long periods of time, even years, solely powered by a battery. Gateways, in turn, are expected to respond $24 / 7$, creating a much higher energy demand and requiring a stable power supply.

The addition of multi-hop capabilities to LoRa or LoRaWAN networks breaks, to some extent, this network model, impacting energy consumption. In general, end nodes now have to spend more time awake, listening to transmissions from other nodes and forwarding packets towards their destination. Some of the surveyed proposals consider this issue and develop strategies to minimize energy consumption (e.g., synchronized, daisy-chain-like wake/sleep cycles) [11], [12], [15]. However, others simply assume devices will be constantly awake and powered, impeding maintenance-less, long-term battery operation [17], [14], [13]. Only one proposal claims that using a mesh topology may reduce power consumption, as using a lower SFs requires less energy per transmitted byte [9].

\section{B. Extending coverage beyond the infrastructure reach}

Some of the reviewed solutions from Section III, such as [8], [9], [10] and, to a lesser extent, [11], [12], [13], and [15], could ease the path for novel developments in the tracking of fleet vehicles. In general, the network topology will vary as the nodes move and the links fluctuate. This dynamicity must be taken into account to adapt forwarding strategies over time. Dealing with networks of different density remains an open challenge, not yet considered by any of the surveyed solutions. On one hand, dense networks could use network segmentation or clustering techniques to limit the scope of the transmissions and achieve a more efficient use of the spectrum. On the other hand, sparse networks, where nodes can remain isolated in partitions for long periods, could benefit from DTN strategies to ensure data eventually reach their destination when the conditions allow for it.

\section{Fault-tolerant systems}

The proposals providing mesh-like capabilities [9], [13], [15], [17] and, to a lesser extent [8], [16], and [10] could provide the starting point for pervasive communications in the aftermath of natural disasters, such as earthquakes, that require some degree of fault-tolerance. The main challenge in this scenario is finding a balance between fast decision-making based on data harvested in the vicinity of the disaster and global planning based on massive, centralized data collection. Other, similar, applications could benefit from the edge computing paradigm since, given the potentially large numbers of messages to spread towards the next hops, data aggregation and filtering are needed to ensure that only significant messages are sent. Additionally, shorter-range technologies (e.g., Wi-Fi, Bluetooth) could be used as "backbones" to bridge LoRa mesh network segments with higher bandwidth or to build a dualstack network. So far, the only proposal combining two radio technologies, [17], uses them with another purpose (Bluetooth for connecting end users' devices and LoRa for long-distance node-to-node communication).

\section{Infrastructure-less systems}

Besides the more generic multi-hop and mesh solutions reviewed in Section III, [16], [17], and [19], there are other 
proposed multi-hop solutions that could be used in deployments where uplink data must be routed towards a central node [9]. A critical issue in this scenario is to ensure that data is securely and privately transmitted to the destination so that attackers cannot tamper with the readings or infer personal information or details from them. Additionally, network fragmentation techniques must be taken into account to ensure the potentially large number of nodes and routes can be managed by devices with low resources.

\section{OPEN CHALLENGES AND OPPORTUNITIES}

The LoRa radio technology has proven to be very successful in IoT scenarios covering areas of tens of square $\mathrm{kms}$, especially as part of the LoRaWAN architecture stack and using a star of stars network topology. We have described three significant scenarios requiring diverse features and capabilities beyond those of LoRaWAN's star of stars, matching them with recent state-of-the-art proposals. Different authors have presented designs and implementations aiming to enhance LoRa and LoRaWAN with multi-hop capability, to extend the coverage of network deployments, or to improve some of their performance aspects. Many works focus on extending the coverage of centralized networks over larger areas, while others pursue flexible mesh topologies, still with a predominant nodes $\rightarrow$ gateway/sink data flow. Fewer proposals aim at decentralized mesh architectures, which could allow for longer-range networks that could better suit applications leveraging computation at the edge.

We have discussed how significant LoRa and LoRaWAN IoT applications could benefit from novel multi-hop and mesh proposals, and we have identified those that could better fit each of the presented scenarios. However, most solutions target very specific problems and are not suitable as generic enablers of multi-hop applications. Therefore, a number of challenges for researchers, developers, and vendors remain open: network scalability with hundreds, or even thousands, of nodes; managing network partitions and complex topologies; combining DTN and opportunistic networking principles; mixing long-range LoRa and short-range Wi-Fi transmissions; energy awareness; security and privacy issues; and others. Also, efforts put into multi-hop and mesh in similar technologies, like 802.15.4, should not be ignored. Indeed, the variety of approaches reveals the importance of enabling LoRa and LoRaWAN with multi-hop to build flexible network topologies, easing the path for novel commercial applications and successful products.

\section{REFERENCES}

[1] K. Mekki, E. Bajic, F. Chaxel, and F. Meyer, "A comparative study of LPWAN technologies for large-scale IoT deployment," ICT express, vol. 5, no. 1, pp. 1-7, 2019. [Online]. Available: https://www.sciencedirect.com/science/article/pii/S2405959517302953

[2] A. Augustin, J. Yi, T. Clausen, and W. M. Townsley, "A study of LoRa: Long range \& low power networks for the Internet of Things," Sensors, vol. 16, no. 9, 2016.

[3] F. Adelantado, X. Vilajosana, P. Tuset-Peiro, B. Martinez, J. MeliaSegui, and T. Watteyne, "Understanding the limits of LoRaWAN," IEEE Commun. Mag., vol. 55, no. 9, pp. 34-40, 2017.

[4] O. Georgiou and U. Raza, "Low power wide area network analysis: Can LoRa scale?" IEEE Wireless Communications Letters, vol. 6, no. 2, pp. 162-165, 2017.

[5] F. Righetti, C. Vallati, D. Comola, and G. Anastasi, "Performance measurements of IEEE $802.15 .4 \mathrm{~g}$ wireless networks," in 2019 IEEE 20th International Symposium on "A World of Wireless, Mobile and Multimedia Networks" (WoWMoM), 2019, pp. 1-6.

[6] J. Haxhibeqiri, E. De Poorter, I. Moerman, and J. Hoebeke, "A survey of LoRaWAN for IoT: From technology to application," Sensors, vol. 18, no. 11, pp. 1-38, 2018. [Online]. Available: https://www.mdpi.com/1424-8220/18/11/3995

[7] W. Shi, J. Cao, Q. Zhang, Y. Li, and L. Xu, "Edge computing: Vision and challenges," IEEE Internet of Things Journal, vol. 3, no. 5, pp. 637-646, 2016.

[8] J. Dias and A. Grilo, "LoRaWAN multi-hop uplink extension," Procedia Comput. Sci., vol. 130, no. C, pp. 424-431, 2018.

[9] B. Sartori, S. Thielemans, M. Bezunartea, A. Braeken, and K. Steenhaut, "Enabling RPL multihop communications based on LoRa," in WiMob, 2017, pp. $1-8$.

[10] D. Lundell, A. Hedberg, C. Nyberg, and E. Fitzgerald, "A routing protocol for LoRa mesh networks," in 2018 IEEE 19th International Symposium on "A World of Wireless, Mobile and Multimedia Networks" (WoWMoM), 2018, pp. 14-19.

[11] C. T. Duong and M. K. Kim, "Multi-hop linear network based on LoRa," Advanced Science and Technology Letters, vol. 150, pp. 29-33, 2018.

[12] A. Abrardo and A. Pozzebon, "A multi-hop LoRa linear sensor network for the monitoring of underground environments: The case of the medieval aqueducts in Siena, Italy," Sensors, vol. 19, no. 2, pp. 1-22, 2019.

[13] H. Lee and K. Ke, "Monitoring of large-area IoT sensors using a LoRa wireless mesh network system: Design and evaluation," IEEE Trans. Instrum. Meas., vol. 67, no. 9, pp. 2177-2187, 2018.

[14] S. Kim, H. Lee, and S. Jeon, "An adaptive spreading factor selection scheme for a single channel LoRa modem," Sensors, vol. 20, no. 4, pp. $1-13,2020$.

[15] M. N. Ochoa, A. Guizar, M. Maman, and A. Duda, "Evaluating LoRa energy efficiency for adaptive networks: From star to mesh topologies," in WiMob, 2017, pp. 1-8.

[16] M. McCauley, RadioHead Packet Radio Library for embedded microprocessors, 2014. [Online]. Available: https://www.airspayce.com/ mikem/arduino/RadioHead/

[17] K. Hester et al., Meshtastic - An opensource hiking, pilot, skiing, secure GPS mesh communicator, 2020. [Online]. Available: https://www.meshtastic.org/

[18] Pycom, Pymesh - LoRa full-mesh network technology., 2020. [Online]. Available: https://docs.pycom.io/pymesh/

[19] NiceRF, SV-MESH Mesh Network Modules, 2020. [Online]. Available: https://www.nicerf.com/products/collections/ uart-transceiver-module.html

[20] J. Petäjäjärvi, K. Mikhaylov, M. Pettissalo, J. Janhunen, and J. Iinatti, "Performance of a low-power wide-area network based on LoRa technology: Doppler robustness, scalability, and coverage," International Journal of Distributed Sensor Networks, vol. 13, no. 3, pp. 1-16, 2017. 


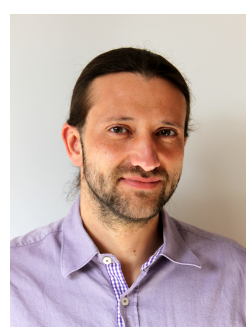

Roger Pueyo Centelles is PhD student at the Distributed Systems Group at Universitat Politècnica de Catalunya (UPC) since 2017. His research interests include Wireless Mesh Community Networks and IoT.

Felix Freitag is Associate Professor at the Computer Architecture Department at UPC. His research interests include Community Networks, Community Clouds with the Cloudy distribution, and the design and evaluation of experimental testbeds.
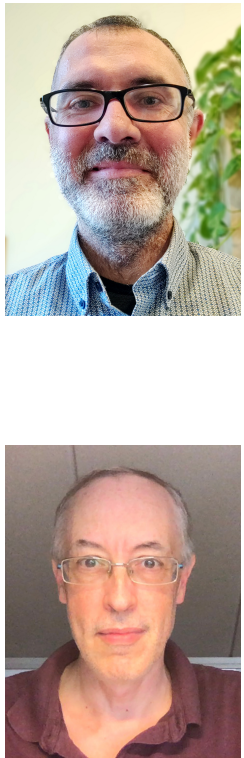

Roc Meseguer is Associate Professor at the Computer Architecture Department at UPC. His research interests include resource allocation for large-scale systems, decentralized systems applied to ambient intelligence and bottom-up networks.

Leandro Navarro is Professor at the Computer Architecture Department at UPC. His research interests include the design of scalable and cooperative Internet-scale and economics-inspired systems. 